\title{
Design and Analysis of Nanoantenna Arrays for Imaging and Sensing Applications at Optical Frequencies
}

\author{
Göktuğ Işıklar, İsa Can Çetin, Mustafa Algun, Özgür Ergül \\ Department of Electrical and Electronics Engineering \\ Middle East Technical University, Ankara, Turkey \\ *corresponding author, E-mail: ozergul@metu.edu.tr
}

\begin{abstract}
We present computational analysis of nanoantenna arrays for imaging and sensing applications at optical frequencies. Arrays of metallic nanoantennas are considered in an accurate simulation environment based on surface integral equations and the multilevel fast multipole algorithm developed for plasmonic structures. Near-zone responses of the designed arrays to nearby nanoparticles are investigated in detail to demonstrate the feasibility of detection. We show that both metallic and dielectric nanoparticles, even with subwavelength dimensions, can be detected.
\end{abstract}

\section{Introduction}

In recent years, the increasing interest in plasmonics has been supported by rapid developments in fabrication techniques, leading to a widespread study of unique characteristics of nanostructures [1]. Thanks to their extensive ability of trapping and focusing electromagnetic waves in subwavelength regions, nanoantennas have been among the most attractive of such metallic structures to manipulate light [2]. Plasmonic nanoantennas can be used in many applications, such as energy harvesting [3, 4], nano-optical focusing $[5,6]$, nano-optical wireless networks $[7,8]$, as well as bio-sensing $[9,10,11,12]$. As shown in this study, nanoantennas can be excellent tools to detect metallic and dielectric nanoparticles for imaging and sensing applications. Specifically, with their strong near-zone coupling properties, nanoantennas may enable tracking nanoparticles, even of subwavelength sizes at optical frequencies. Since optimal arrangements of nanoantennas in terms of cost, detection reliability, and fabrication feasibility are not well known, extensive studies are needed to design nanoantenna arrays for effective nanoparticle detection.

Optimal arrangements of nanoantennas strongly depend on the selected shape, size, and material, as well as the operating frequency. Due to their easier fabrication, basic bowtie structures are frequently used in the literature. In fact, despite their relatively simple geometries, bowtie nanoantennas can create intense hot spots at their feed gaps [13]. The chosen material and the size of nanoantennas are responsible for the resonance frequency range, where electromagnetic fields are strongly focused. In addition, the periodicity in the arrangements of multiple nanoantennas is one of the most important factors when designing effective arrays for detection. This study is devoted to the design of such arrays that have distinguishable responses to nearby particles. Once a nanoantenna design is fixed based on the geometry and material, we investigate distances between nanoantennas to construct small-scale arrays. Then, the electromagnetic responses of the designed arrays to different particles in diverse scenarios are rigorously investigated using a very accurate simulation environment.

Their sensitive responses to electromagnetic waves make nanoantennas challenging to analyze via computational methods [14]. In this work, the fast and accurate implementation to analyze nanoantenna arrays and nearby particles is based on a modified combined tangential (MCTF) formulation $[15,16]$ in the frequency domain. The effective complex permittivity values for metals are extracted from Lorentz-Drude models or measurement results [17]. Constructed matrix equations via discretization are solved by using the multilevel fast multipole algorithm (MLFMA) [18] that is developed for plasmonic media [19]. By means of accurate simulations, various imaging and sensing scenarios are investigated in detail to demonstrate the feasibility of particle detection with nanoantenna arrays.

In the next section, we present the details of the numerical solver. Then, Section 3 includes the design of nanoantenna arrays for particle detection, followed by numerical results in Section 4 and concluding remarks in Section 5.

\section{Accurate Simulation Environment}

Electromagnetic problems involving nanoantenna arrays and particles (to be detected) are solved in the frequency domain. We particularly use MCTF [15] that is suitable to analyze metallic objects in wide ranges of optical frequencies. MCTF is discretized with the Rao-Wilton-Glisson (RWG) functions, leading to dense matrix equations. For a composite problem involving $U+1$ regions labeled as $D_{u}$ for $u=0,1, \cdots, U$, including the host medium (vacuum) $D_{0}$, matrix equations can be written as

$$
\left[\begin{array}{ll}
\overline{\boldsymbol{Z}}_{11} & \overline{\boldsymbol{Z}}_{12} \\
\overline{\boldsymbol{Z}}_{21} & \overline{\boldsymbol{Z}}_{22}
\end{array}\right] \cdot\left[\begin{array}{l}
\boldsymbol{x} \\
\boldsymbol{y}
\end{array}\right]=\left[\begin{array}{c}
\boldsymbol{v} \\
\boldsymbol{w}
\end{array}\right]
$$




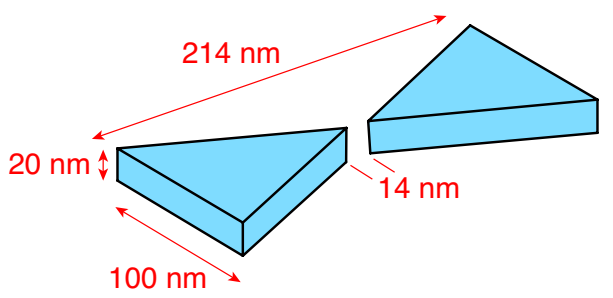

(a)
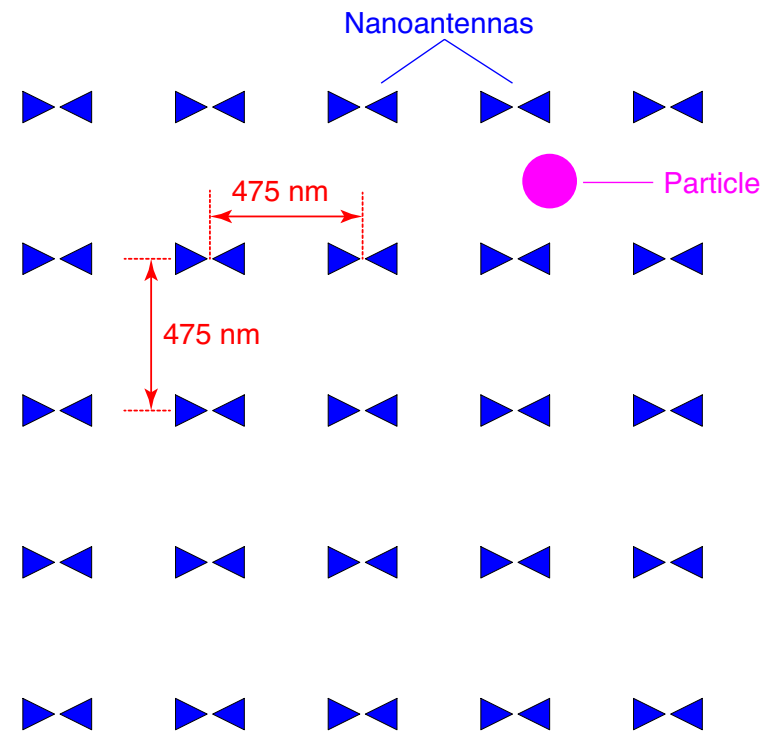

(b)

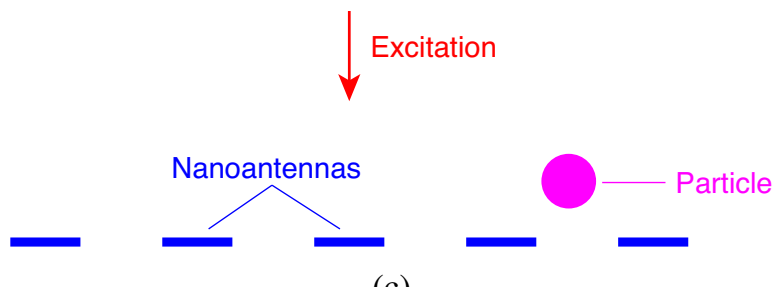

(c)

Figure 1: (a) The bowtie geometry for nanoantennas considered in this paper. (b) An arrangement of $5 \times 5$ nanoantennas with $475 \mathrm{~nm}$ periodicity and a spherical particle located in the vicinity. (c) Side view of the $5 \times 5$ array and the particle located above it.

where

$$
\begin{aligned}
\overline{\boldsymbol{Z}}_{11} & =\sum_{u=0}^{U} \eta_{u} \overline{\boldsymbol{T}}_{u}, \quad \overline{\boldsymbol{Z}}_{12}=-\sum_{u=0}^{U} \overline{\boldsymbol{K}}_{u} \\
\overline{\boldsymbol{Z}}_{21} & =\sum_{u=0}^{U} \eta_{u} \eta_{u^{\prime}} \overline{\boldsymbol{K}}_{u}, \quad \overline{\boldsymbol{Z}}_{22}=\sum_{u=0}^{U} \eta_{u^{\prime}} \overline{\boldsymbol{T}}_{u} \\
\boldsymbol{v} & =\sum_{u=0}^{U} \boldsymbol{v}_{u}, \quad \boldsymbol{w}=\sum_{u=0}^{U} \boldsymbol{w}_{u} .
\end{aligned}
$$

In the above, $\boldsymbol{x}$ and $\boldsymbol{y}$ are arrays of expansion coefficients for equivalent electric and magnetic currents, respectively, while $\eta_{u}=\sqrt{\mu_{u}} / \sqrt{\epsilon_{u}}$ is the characteristic impedance of medium $u$. In addition, $u^{\prime}$ represents the domain that is

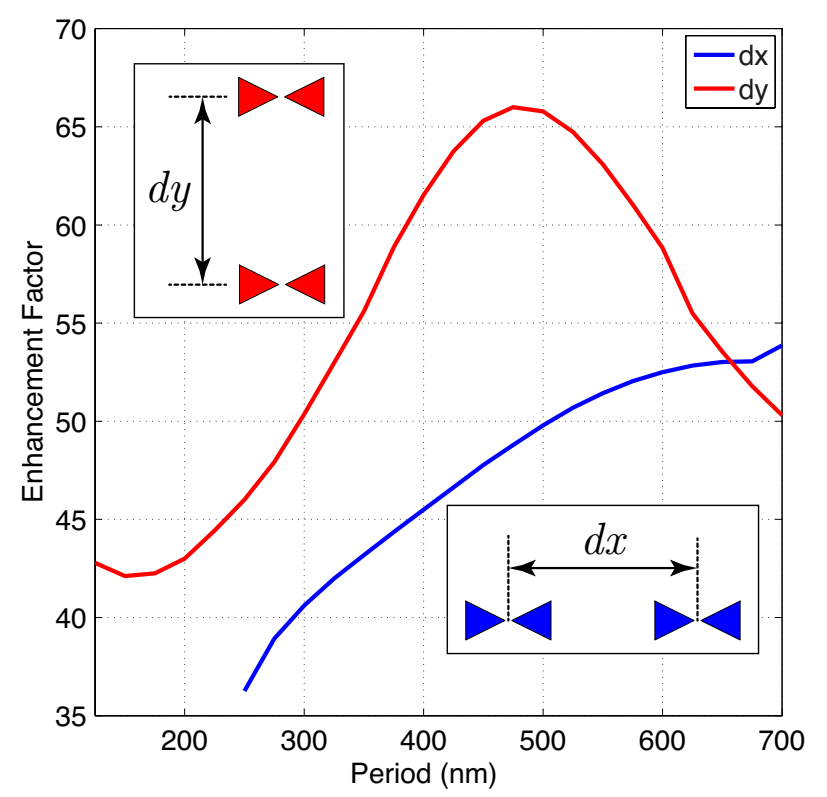

Figure 2: Power enhancement factor at the gap of an Ag bowtie nanoantenna, when another identical nanoantenna is placed at different nearby locations.

sharing the interface with domain $u$, at which the testing operation is applied. Electromagnetic interactions (discretized operators) are derived as

$$
\begin{aligned}
& \overline{\boldsymbol{T}}_{u}[m, n]=\xi_{m, u} \xi_{n, u} \int_{S_{m}} d \boldsymbol{r} \boldsymbol{t}_{m}(\boldsymbol{r}) \cdot \mathcal{T}_{u}\left\{\boldsymbol{b}_{n}\right\}(\boldsymbol{r}) \\
& \overline{\boldsymbol{K}}_{u}[m, n]=\xi_{m, u} \xi_{n, u} \int_{S_{m}} d \boldsymbol{r} \boldsymbol{t}_{m}(\boldsymbol{r}) \cdot \mathcal{K}_{u}\left\{\boldsymbol{b}_{n}\right\}(\boldsymbol{r}),
\end{aligned}
$$

where $\boldsymbol{t}_{m}$ and $\boldsymbol{b}_{n}$ represent testing and basis functions, $\xi_{n, u}, \xi_{m, u}= \pm 1$ represent orientations of testing/basis functions with respect to domain $u$, while the integrodifferential operators can be written as

$$
\begin{aligned}
\mathcal{T}_{u}\{\boldsymbol{X}\}(\boldsymbol{r}) & =i k_{u} \int d \boldsymbol{r}^{\prime} \boldsymbol{X}\left(\boldsymbol{r}^{\prime}\right) g_{u}\left(\boldsymbol{r}, \boldsymbol{r}^{\prime}\right) \\
& +\frac{i}{k_{u}} \int d \boldsymbol{r}^{\prime} \nabla^{\prime} \cdot \boldsymbol{X}\left(\boldsymbol{r}^{\prime}\right) \nabla g_{u}\left(\boldsymbol{r}, \boldsymbol{r}^{\prime}\right) \\
\mathcal{K}_{u}\{\boldsymbol{X}\}(\boldsymbol{r}) & =\int_{\mathrm{PV}} d \boldsymbol{r}^{\prime} \boldsymbol{X}\left(\boldsymbol{r}^{\prime}\right) \times \nabla^{\prime} g_{u}\left(\boldsymbol{r}, \boldsymbol{r}^{\prime}\right) .
\end{aligned}
$$

These operators involve the Green's function $g_{u}\left(\boldsymbol{r}, \boldsymbol{r}^{\prime}\right)=$ $\exp \left(i k_{u}\left|\boldsymbol{r}-\boldsymbol{r}^{\prime}\right|\right) /\left(4 \pi\left|\boldsymbol{r}-\boldsymbol{r}^{\prime}\right|\right)$ and the wavenumber $k_{u}=$ $\omega \sqrt{\mu} \sqrt{\epsilon}$ with angular frequency $\omega$. Finally, contributions to excitation vectors for MCTF are derived as

$$
\begin{aligned}
\boldsymbol{v}_{u} & =-\xi_{m, u} \int_{S_{m}} d \boldsymbol{r} \boldsymbol{t}_{m}(\boldsymbol{r}) \cdot \boldsymbol{E}_{u}^{\mathrm{inc}}(\boldsymbol{r}) \\
\boldsymbol{w}_{u} & =-\eta_{u} \eta_{u^{\prime}} \xi_{m, u} \int_{S_{m}} d \boldsymbol{r} \boldsymbol{t}_{m}(\boldsymbol{r}) \cdot \boldsymbol{H}_{u}^{\mathrm{inc}}(\boldsymbol{r}),
\end{aligned}
$$

where $\boldsymbol{E}_{u}^{\mathrm{inc}}(\boldsymbol{r})$ and $\boldsymbol{H}_{u}^{\mathrm{inc}}(\boldsymbol{r})$ represent the incident electric/magnetic field intensity created by external sources located inside $D_{u}$. 

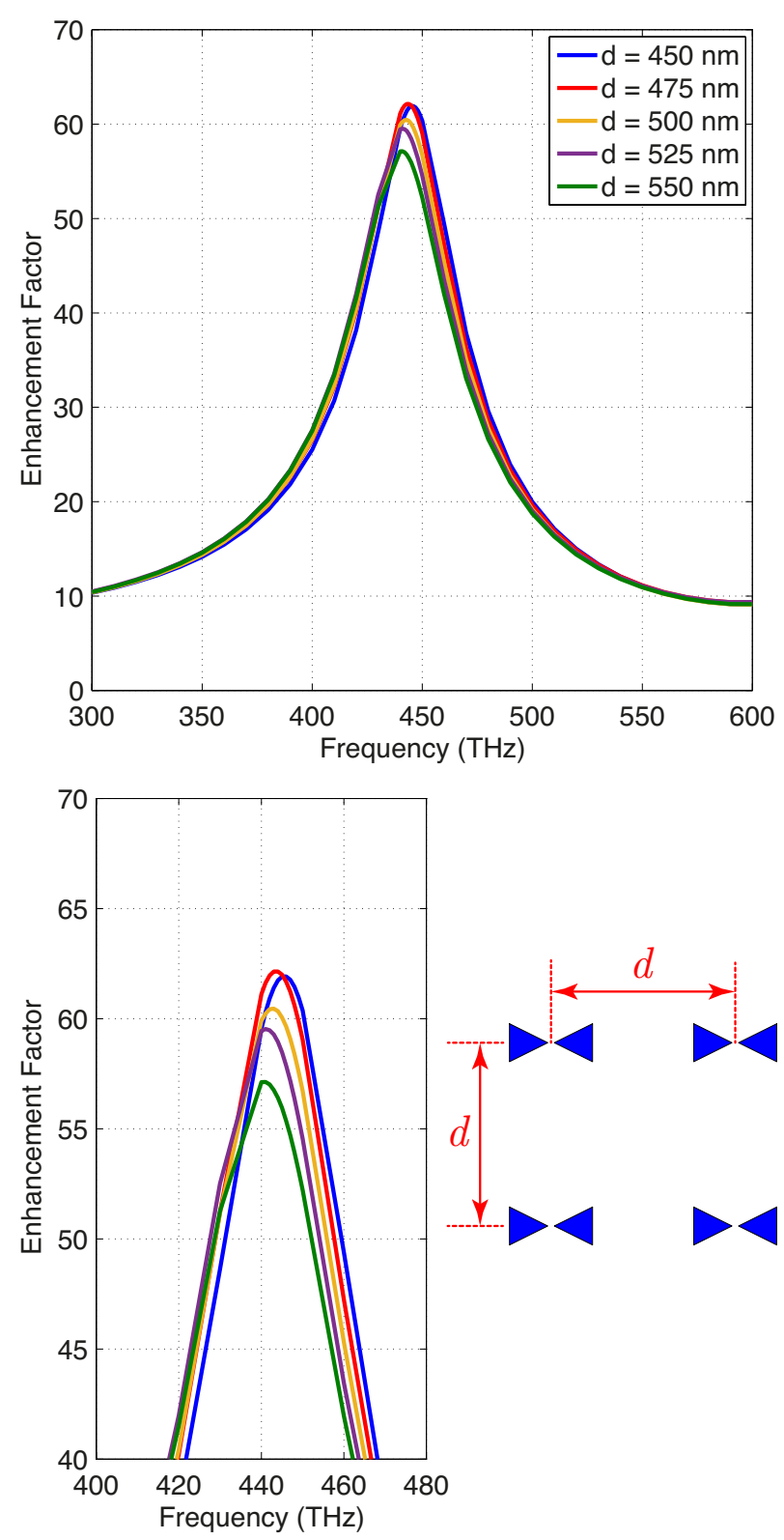

Figure 3: Power enhancement factor at the gap of an $\mathrm{Ag}$ bowtie nanoantenna in an arrangement of $2 \times 2$ nanoantennas with different periodicities.

In the examples of this paper, we consider nanoantenna arrays, typically made of Ag. The complex permittivity of $\mathrm{Ag}$ depending on the frequency is extracted from the Lorentz-Drude model. In some cases, the particle to be detected is also $\mathrm{Ag}$, leading to a problem with $U=1$ to be formulated with MCTF as in [15]. In other cases, dielectric particles are considered with a fixed permittivity, leading to three-region composite problems $(U=2)$. The developed formulation allows the use of $U>2$, while such cases are beyond the scope of this paper.

Matrix equations derived from the discretization of MCTF are solved iteratively by using MLFMA. For interactions inside metallic objects that are modeled as pene-

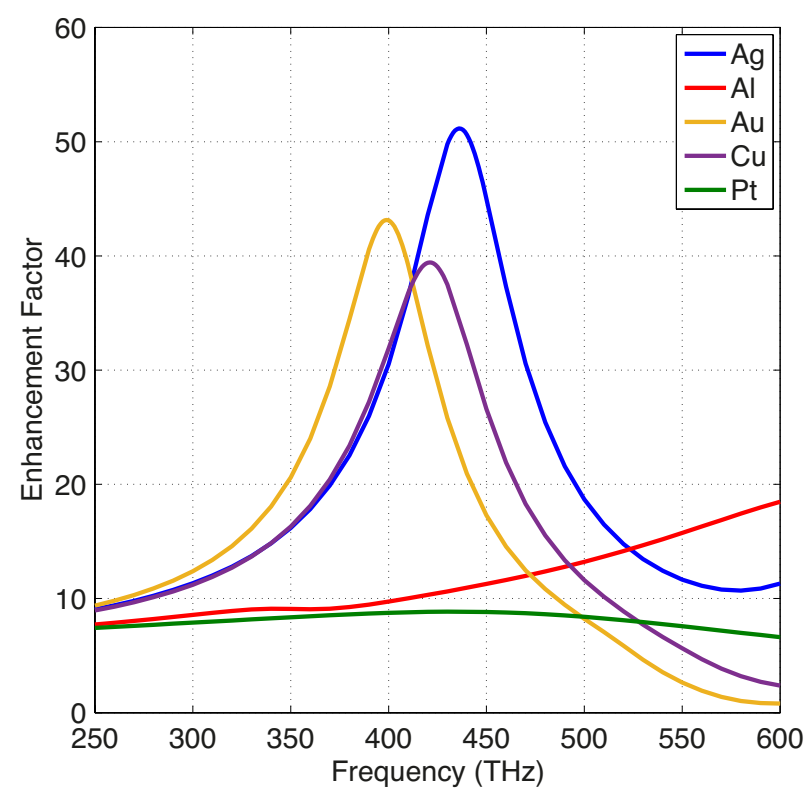

Figure 4: Power enhancement factor at the gap of an bowtie nanoantenna when different materials are used.

trable bodies, interaction truncation is applied as described in [19]. Once the expansion coefficients are found, the electric field intensity and the magnetic field intensity at an observation point $\boldsymbol{r}$ located inside $D_{u}$ are computed as

$$
\begin{aligned}
\boldsymbol{E}(\boldsymbol{r}) & =\boldsymbol{E}_{u}^{\mathrm{inc}}(\boldsymbol{r}) \\
& +i k_{u} \eta_{u} \sum_{n} \boldsymbol{x}[n] \xi_{n, u} \int_{S_{n}} d \boldsymbol{r}^{\prime} \boldsymbol{b}_{n}\left(\boldsymbol{r}^{\prime}\right) g_{u}\left(\boldsymbol{r}, \boldsymbol{r}^{\prime}\right) \\
& -i \frac{\eta_{u}}{k_{u}} \sum_{n} \boldsymbol{x}[n] \xi_{n, u} \int_{S_{n}} d \boldsymbol{r}^{\prime} \nabla^{\prime} \cdot \boldsymbol{b}_{n}\left(\boldsymbol{r}^{\prime}\right) \nabla^{\prime} g_{u}\left(\boldsymbol{r}, \boldsymbol{r}^{\prime}\right) \\
& -\sum_{n} \boldsymbol{y}[n] \xi_{n, u} \int_{S_{n}} d \boldsymbol{r}^{\prime} \boldsymbol{b}_{n}\left(\boldsymbol{r}^{\prime}\right) \times \nabla^{\prime} g_{u}\left(\boldsymbol{r}, \boldsymbol{r}^{\prime}\right)
\end{aligned}
$$

and

$$
\begin{aligned}
\boldsymbol{H}(\boldsymbol{r}) & =\boldsymbol{H}_{u}^{\mathrm{inc}}(\boldsymbol{r}) \\
& +\frac{i k_{u}}{\eta_{u}} \sum_{n} \boldsymbol{y}[n] \xi_{n, u} \int_{S_{n}} d \boldsymbol{r}^{\prime} \boldsymbol{b}_{n}\left(\boldsymbol{r}^{\prime}\right) g_{u}\left(\boldsymbol{r}, \boldsymbol{r}^{\prime}\right) \\
& -\frac{i}{k_{u} \eta_{u}} \sum_{n} \boldsymbol{y}[n] \xi_{n, u} \int_{S_{n}} d \boldsymbol{r}^{\prime} \nabla^{\prime} \cdot \boldsymbol{b}_{n}\left(\boldsymbol{r}^{\prime}\right) \nabla^{\prime} g_{u}\left(\boldsymbol{r}, \boldsymbol{r}^{\prime}\right) \\
& +\sum_{n} \boldsymbol{x}[n] \xi_{n, u} \int_{S_{n}} d \boldsymbol{r}^{\prime} \boldsymbol{b}_{n}\left(\boldsymbol{r}^{\prime}\right) \times \nabla^{\prime} g_{u}\left(\boldsymbol{r}, \boldsymbol{r}^{\prime}\right)
\end{aligned}
$$

where summations include all basis functions $\boldsymbol{b}_{n}$ located on the surface of the domain. Then, the power density is obtained as

$$
\boldsymbol{S}(\boldsymbol{r})=\boldsymbol{E}(\boldsymbol{r}) \times[\boldsymbol{H}(\boldsymbol{r})]^{*},
$$

where ${ }^{*}$ represents complex conjugate. We also compute pointwise enhancement factor (scaled power density) as

$$
e(\boldsymbol{r})=\frac{|\boldsymbol{S}(\boldsymbol{r})|}{\left|\boldsymbol{S}_{u}^{\text {inc }}(\boldsymbol{r})\right|}=\frac{|\boldsymbol{S}(\boldsymbol{r})|}{\left|\boldsymbol{E}_{u}^{\text {inc }}(\boldsymbol{r}) \times\left[\boldsymbol{H}_{u}^{\text {inc }}(\boldsymbol{r})\right]^{*}\right|} .
$$


ELECTRIC FIELD INTENSITY
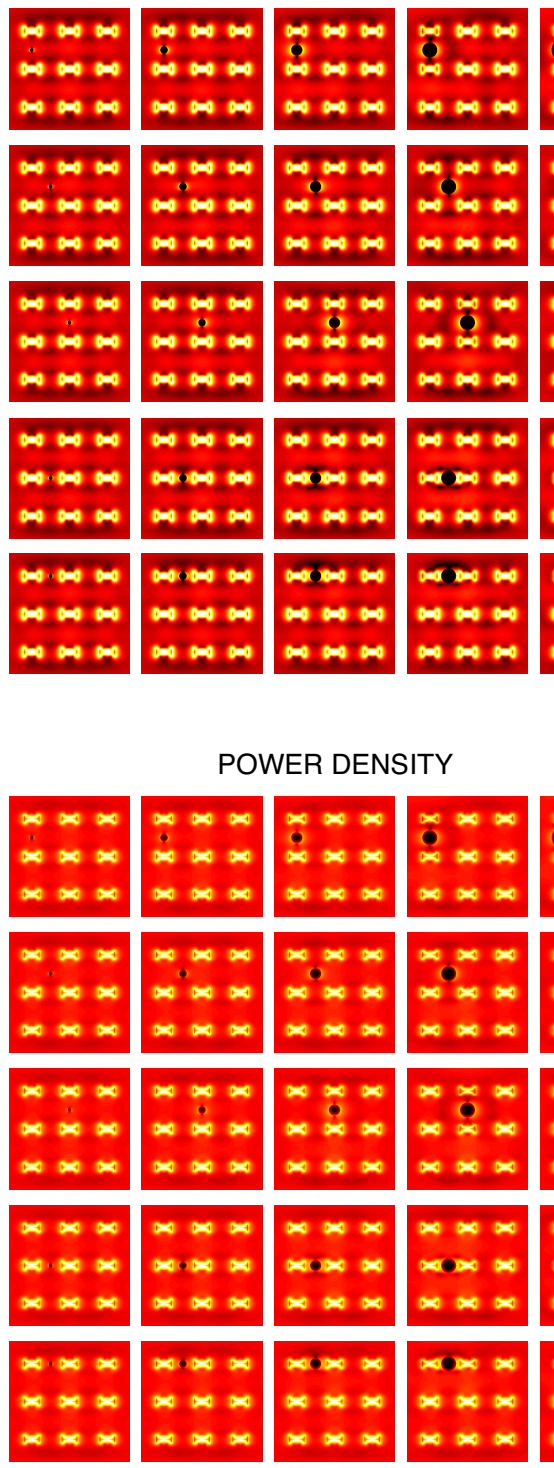

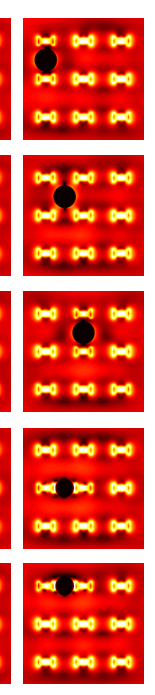

POWER DENSITY
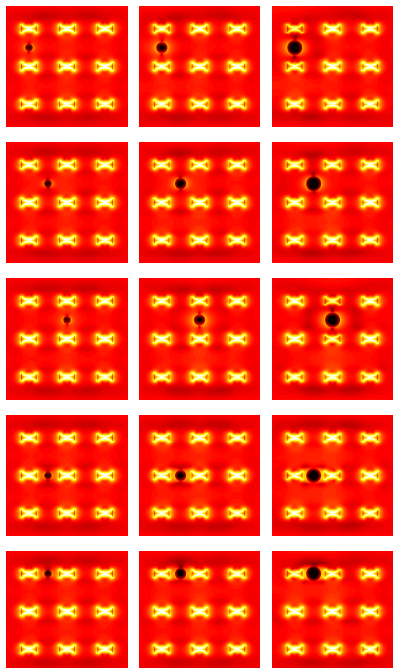

$20 \mathrm{dBV} / \mathrm{m}$

15

10

5

0

$-5$

$-5$

$-10$

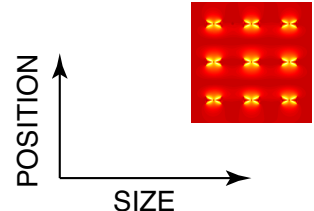

SIZE

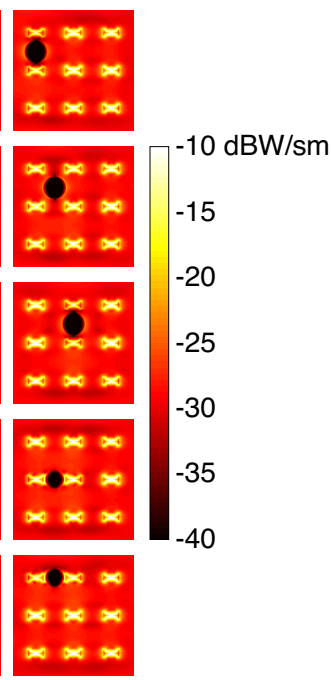

MAGNETIC FIELD INTENSITY

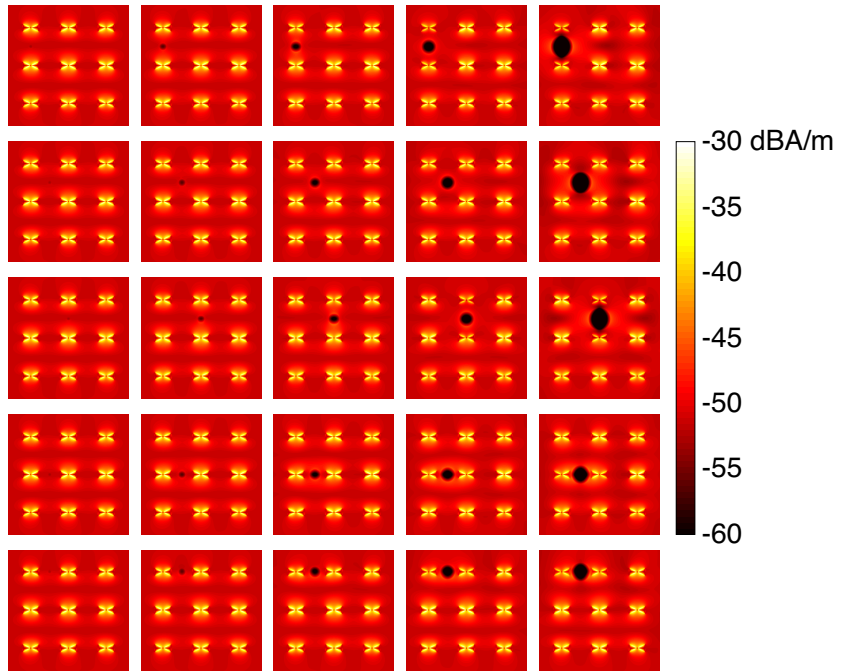

ENHANCEMENT
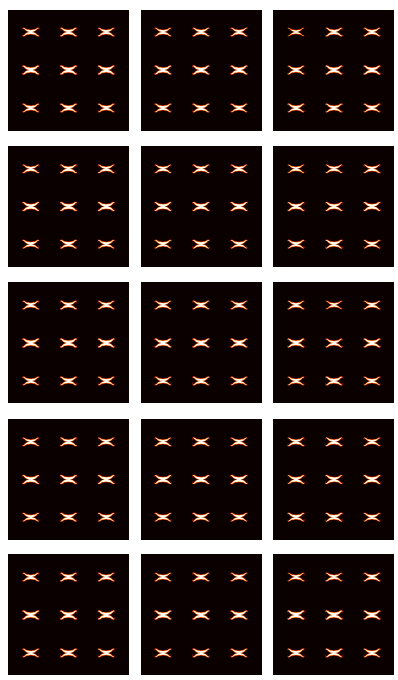
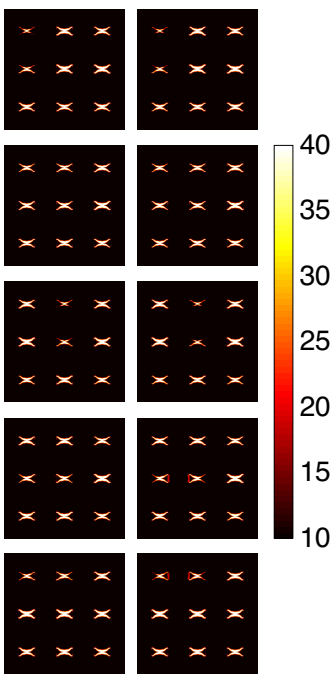

Figure 5: Near-zone characteristics of an array of $3 \times 3$ nanoantennas when Ag particles with different sizes are located in the array plane. The periodicity of the array is $500 \mathrm{~nm}$, while the particle diameter changes from $50 \mathrm{~nm}$ to $300 \mathrm{~nm}$ (for the first three locations) or from $50 \mathrm{~nm}$ to $250 \mathrm{~nm}$ (for the last two locations).

As a typical excitation, we use $1 \mathrm{~V} / \mathrm{m}$ plane wave in the host medium (vacuum $D_{0}$ ), which corresponds to the incident power density value as $\left|\boldsymbol{S}_{0}^{\text {inc }}(\boldsymbol{r})\right| \approx 2.65 \times 10^{-3} \mathrm{~W} / \mathrm{sm}$.

\section{Nanoantenna Arrays for Particle Detection}

In this paper, we consider arrays of nanoantennas particularly with bowtie shapes as the most common types used in the literature. With the dimensions given in Fig. 1(a), these structures exhibit relatively high power enhancement factors (if suitable metals are used) in the $400-500 \mathrm{THz}$ range. We arrange multiple nanoantennas, often periodically with a fixed distance between consecutive elements, leading to two-dimensional arrays. For example, a $5 \times 5$ arrangement is depicted in Fig. 1(b) when the periodicity is $475 \mathrm{~nm}$. As also shown in Figs. 1(b) and (c), a particle to be detected is located in the vicinity of the array, while the entire structure is excited by a light source (modeled as a plane wave) from the top.

\subsection{Periodicity of Elements}

Since array elements (bowtie nanoantennas) strongly interact with each other, distances between them are critical for their performances. Placing nanoantennas far away from each other would reduce the interaction between them, while this decreases the reliability of the particle detection, especially for small-scale objects. In fact, electromagnetic interactions between nanoantennas can be employed in a useful way to improve the performances of individual elements. As an example, Fig. 2 presents the power enhancement factor at the gap of an $\mathrm{Ag}$ bowtie nanoantenna, when 


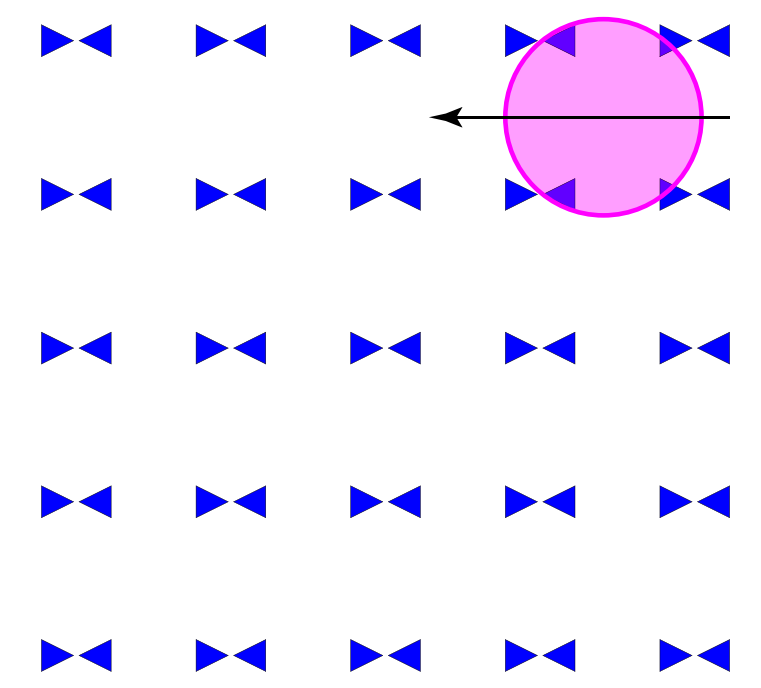

(a)

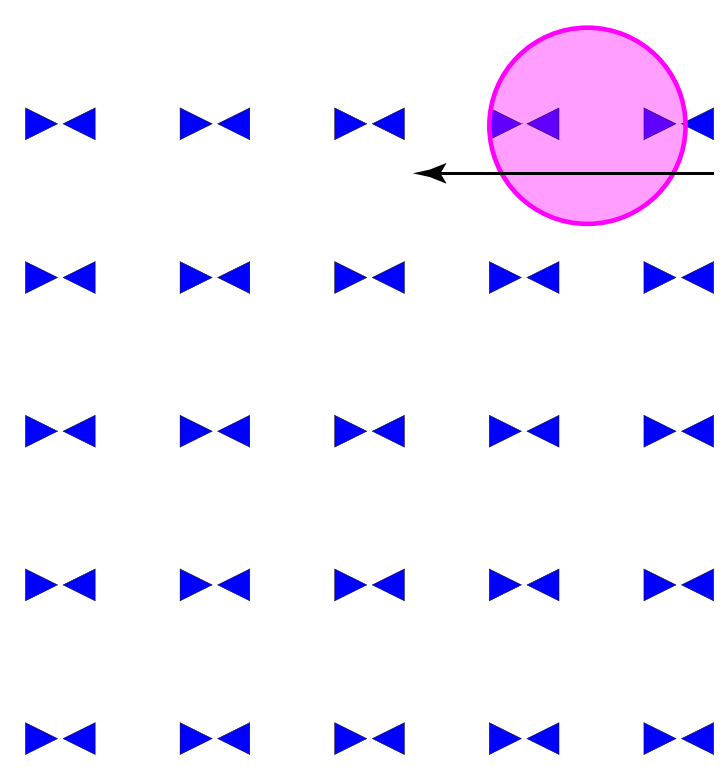

(b)

Figure 6: Movement of a spherical particle of diameter $600 \mathrm{~nm}$ above a $5 \times 5$ array of nanoantennas with $475 \mathrm{~nm}$ periodicity. (a) Moving on a path between two rows. (b) Moving on a path above the first row of nanoantennas.

another identical nanoantenna is placed at different locations. The results show that the enhancement factor is quite sensitive to the distance between the nanoantennas when they are vertically aligned. Specifically, the enhancement factor can exceed 65 , which is higher than the enhancement by a single nanoantenna (50-55), when the centerto-center distance is $475 \mathrm{~nm}$. It is remarkable that placing nanoantennas horizontally always deteriorates the enhancement factor, which must be considered in two-dimensional arrangements. As shown in Fig. 3 for a $2 \times 2$ array of $\mathrm{Ag}$ nanoantennas, the enhancement factor can be kept above 60 at around $445 \mathrm{THz}$, if the periodicity (in horizontal and vertical directions) is fixed to $450-500 \mathrm{~nm}$.

\subsection{Material of Nanoantennas}

As it is well known, the material type is a crucial factor for the enhancement ability of a nanoantenna. For the nanoantenna geometry depicted in Fig. 1(a), the effect of the material is briefly considered in Fig. 4. The enhancement factor is found at the gap of a single nanoantenna, when the material is selected as $\mathrm{Ag}, \mathrm{Al}, \mathrm{Au}, \mathrm{Cu}$, and Pt. Without strong plasmonic responses, $\mathrm{Al}$ and $\mathrm{Pt}$ are not very suitable to obtain high power enhancement factors, while the best performance in terms of achievable maximum enhancement is obtained with Ag. We note that, Au may be preferred depending on the frequency, e.g., it provides more than 40 enhancement factor at $400 \mathrm{THz}$, while the corresponding value is around 30 if $\mathrm{Ag}$ is used at the same frequency.

\section{Detection of Particles}

After numerous numerical analysis, some of which are shown above, the bowtie geometry in Fig. 1(a) is used in array configurations for the detection of particles. The material is selected as Ag, while the detection is considered at $450 \mathrm{THz}$, i.e., when the wavelength in the host medium (vacuum) is approximately $666.2 \mathrm{~nm}$. The periodicity in array configurations is selected as $475-500 \mathrm{~nm}$.

\subsection{Effect of Nearby Particles}

As the first set of results about particle detection, we consider spherical Ag particles with different diameters from $50 \mathrm{~nm}$ to $300 \mathrm{~nm}$. The particles are located in the plane of a $3 \times 3$ nanoantenna array, as depicted in Fig. 5. In addition to different sizes, we consider different locations for the particles in order to investigate their effects in the near-zone characteristics. Fig. 5 presents the electric field intensity $([-10,20] \mathrm{dBV} / \mathrm{m}$ range $)$, the magnetic field intensity $([-60,-30] \mathrm{dBA} / \mathrm{m}$ range $)$, and the power density $([-40,-10] \mathrm{dBW} / \mathrm{sm}$ range), as well as the pointwise power enhancement factor distributions for 25 different cases. The near-zone samples are selected in the array plane $(z=0)$ so that the particles are also seen as dark circles, while this does not directly mean detection since the sampling plane (that cuts the particles) may not be realistic for a detection application. On the other hand, our aim here to observe the response of nanoantennas to the existence of particles. We note that the smallest particle with $50 \mathrm{~nm}$ diameter does not lead to any visible response (first column in each panel), while near-zone field and density distributions significantly change as the particle size grows. This is particularly seen in the near-zone electric field intensity plots, where the coupling between the particles and nearby nanoantennas is observed as relatively large fields. In the power enhancement plots, nanoparticles lead to decreased enhancement values (faded hot spots), which can be interpreted as shadowing depending on the particle size. 


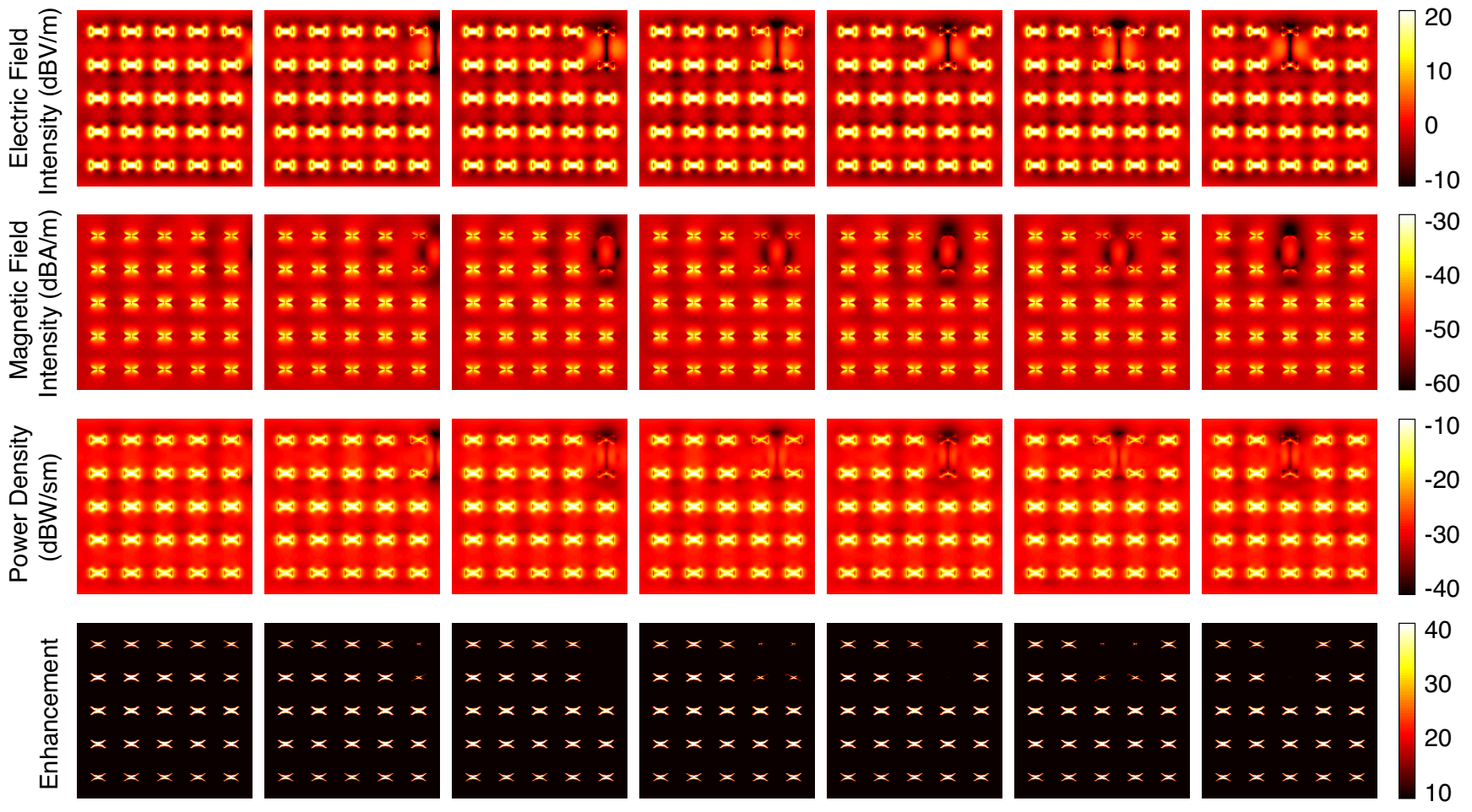

Figure 7: Near-zone characteristics of an array of $5 \times 5$ nanoantennas when an Ag particle of diameter $600 \mathrm{~nm}$ moves above the array as described in Fig. 6(a). The periodicity of the array is $475 \mathrm{~nm}$. The bottom of the particle is $10 \mathrm{~nm}$ away from the top surface of the nanoantennas.

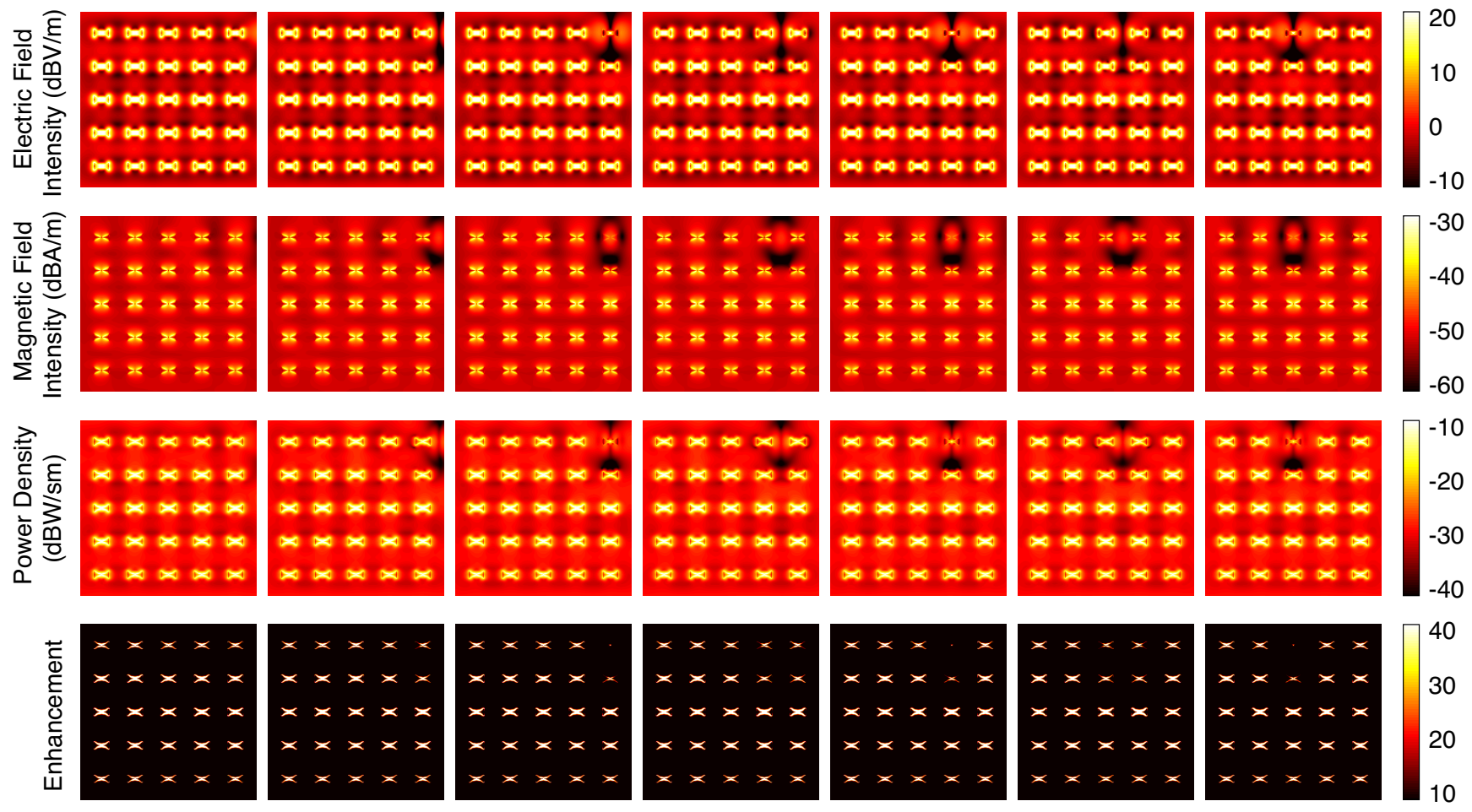

Figure 8: Near-zone characteristics of an array of $5 \times 5$ nanoantennas when an Ag particle of diameter $600 \mathrm{~nm}$ moves above the array as described in Fig. 6(b). The periodicity of the array is $475 \mathrm{~nm}$. The bottom of the particle is $10 \mathrm{~nm}$ away from the top surfaces of the nanoantennas. 

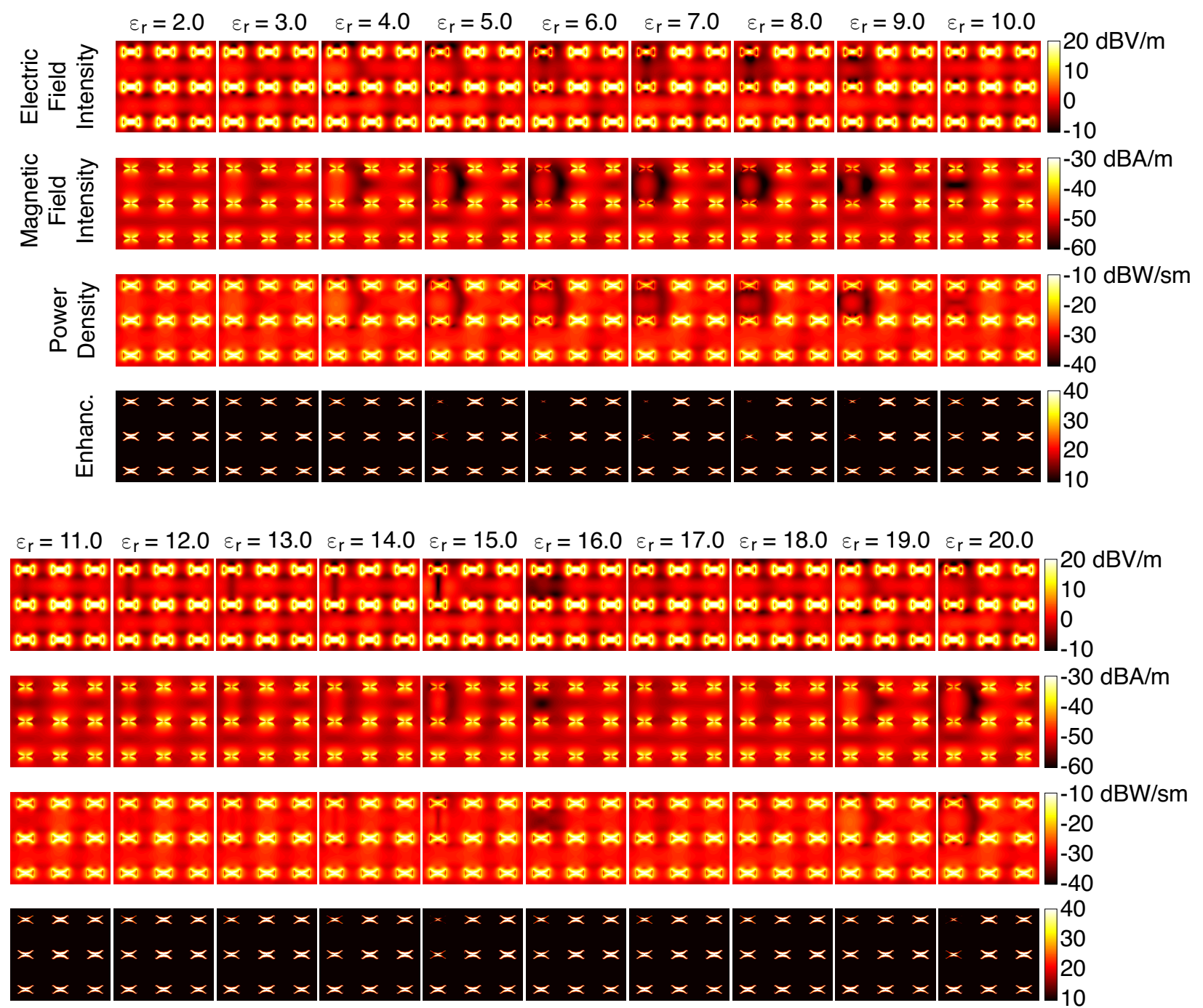

Figure 9: Near-zone characteristics of an array of $3 \times 3$ nanoantennas when dielectric particles of diameter $300 \mathrm{~nm}$ are located above the array. The periodicity of the array is $475 \mathrm{~nm}$. The particles are $90 \mathrm{~nm}$ away from the top surfaces of the nanoantennas.

\subsection{Detection of Moving Particles}

Next, we consider more realistic cases, where a spherical particle is moving above a $5 \times 5$ array of nanoantennas. Fig. 6 presents two different scenarios for the movement of a spherical Ag particle of diameter $600 \mathrm{~nm}$. The bottom of the sphere is $10 \mathrm{~nm}$ away from nanoantenna surfaces. Fig. 7 presents the electric field intensity, the magnetic field intensity, the power density, and the pointwise enhancement factor in the array plane, when the particle moves as depicted in Fig. 6(a). The movement of the particle is clearly observed as deteriorated near-zone distributions, particularly as shadowed nanoantennas. This shadowing effect is significant especially in the enhancement plots, where dark areas occur depending in the location of the particle. Fig. 8 presents similar results when the movement of the particle is as described in Fig. 6(b). In addition to the successful tracking of the particle, it is remarkable that the results in Fig. 8 differ from those in Fig. 7, i.e., the vertical location of the particle can also be identified from near-zone distributions.

\subsection{Detection of Dielectric Particles}

The results so far involve metallic particles, specifically made of $\mathrm{Ag}$, which are generally easier to detect. As the next set of results, we consider dielectric particles with various permittivity values. Fig. 9 presents intensity, density, and enhancement distributions in the array plane when spherical dielectric particles of diameter $300 \mathrm{~nm}$ are located above a $3 \times 3$ array of nanoantennas. The distance between spheres and nanoantenna surfaces is set to $90 \mathrm{~nm}$. The relative permittivity $\left(\varepsilon_{r}\right)$ is changed from 2.0 to 20.0. We observe that the sphere with 2.0 relative permittivity has little effect on the near-zone distributions, while the detection becomes easier as the relative permittivity increases towards 9.0. Interestingly, the effect of the particle is not significant for some higher contrasts, particularly 


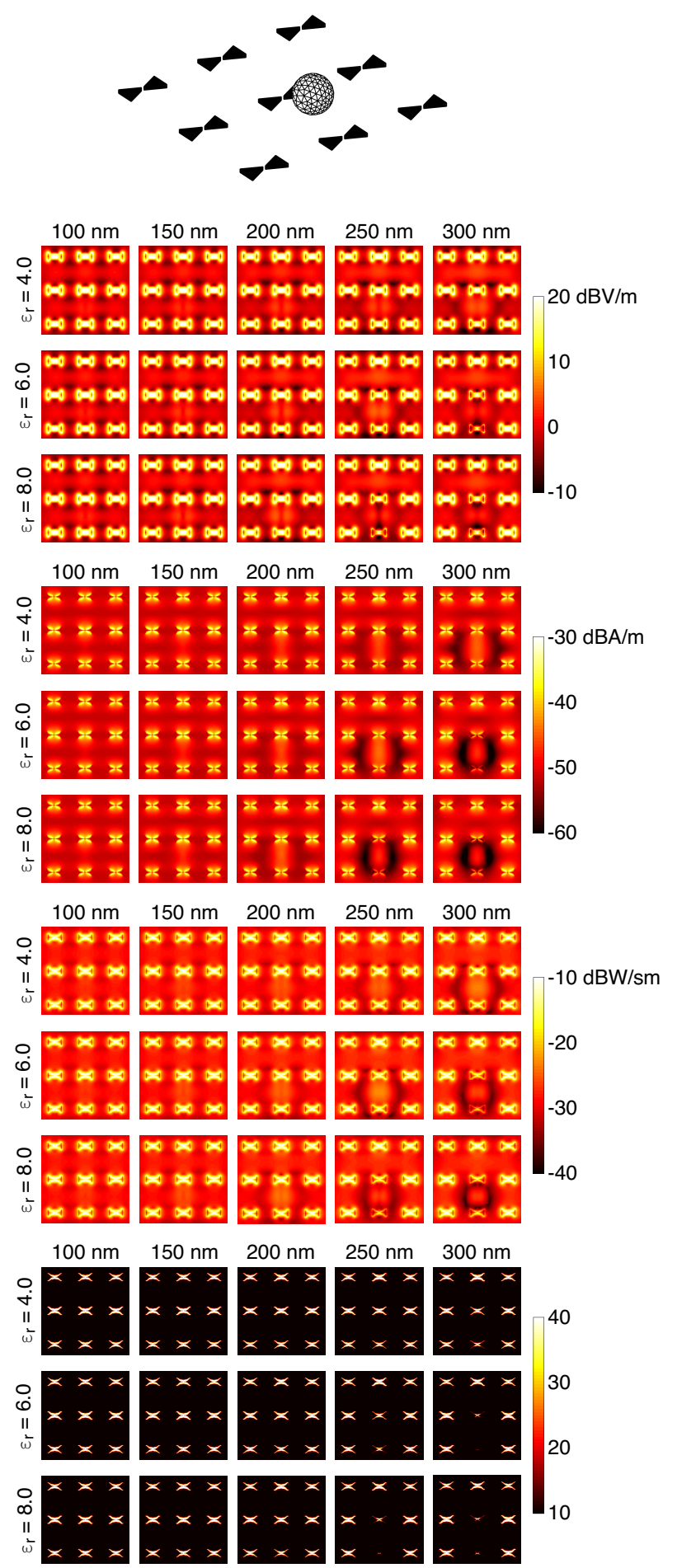

Figure 10: Near-zone characteristics of an array of $3 \times 3$ nanoantennas when dielectric particles with different sizes (25-300 nm diameter) and permittivity values of 4.0,6.0, and 8.0 are located above the array. The distance between the particles and surfaces of nanoantennas is $20 \mathrm{~nm}$.

when $\varepsilon_{r}=11.0-13.0$ and $\varepsilon_{r}=17.0-19.0$. Hence, higher permittivity/contrast values do not necessarily indicate easier detection. Fig. 10 presents another set of results, where particles with different sizes and permittivity values of 4.0,
6.0, and 8.0 are considered. Specifically, in these results, the diameter changes from $100 \mathrm{~nm}$ to $300 \mathrm{~nm}$, while the distance between spherical particles and nanoantenna surfaces is selected as $20 \mathrm{~nm}$. The results show that the detection is easier when the particle size $200 \mathrm{~nm}$ or larger for all three permittivity values.

\subsection{Visualization at Other Planes}

As shown above, some dielectric particles, even with relatively high contrasts, may not be easily detected by considering field and density distributions in the array plane. While this is related to particle size and contrast, as well as particle-to-array distance, numerical investigations show that the relationship is not trivial. On the other hand, one may consider other planes, particularly the plane of incidence, to increase the reliability of detection. As an example, Fig. 11 presents field, density, and pointwise enhancement distributions for spherical particles of diameter $300 \mathrm{~nm}$ with relative permittivity values of $3.0,6.0$, and 10.0. In the plane of array, which is now called Plane 1, the particle with $\varepsilon=6.0$ is easily detected, while the others have limited effects on the near-zone characteristics. In the plane of incidence (Plane 2), however, particles lead to unique distributions (except for the enhancement plots) such that all of them become detectable. We emphasize that distributions outside the particles should be considered for detection purposes since sampling inside particles may not be possible depending on the application.

\subsection{Geometric Identification}

In many applications, not only the existence but also the shape of nanoparticles is critical to be detected. This can be possible if the obtained near-zone distributions are compared with reference results in a database. In order to demonstrate the feasibility of geometric identification, Fig. 12 shows near-zone distributions when dielectric particles with different shapes exist above a $3 \times 3$ array of nanoantennas. The relative permittivity of the particles is set to 8.0. In each case, the distance between the particle and the array surface is $50 \mathrm{~nm}$. The results in Fig. 12 show that, despite similarities, particles lead to different field, density, and/or enhancement distributions. This means that geometric identification can be possible even with arrays involving small numbers of nanoantennas.

\section{Concluding Remarks}

In this paper, design and computational analysis of nanoantenna arrays for particle detection applications are presented. By using a highly accurate simulation environment, alternative nanoantenna arrays and nearby nanoparticles are investigated in diverse scenarios. The results demonstrate the feasibility of sensing and imaging of metallic and dielectric particles, even of subwavelength sizes at optical frequencies. 

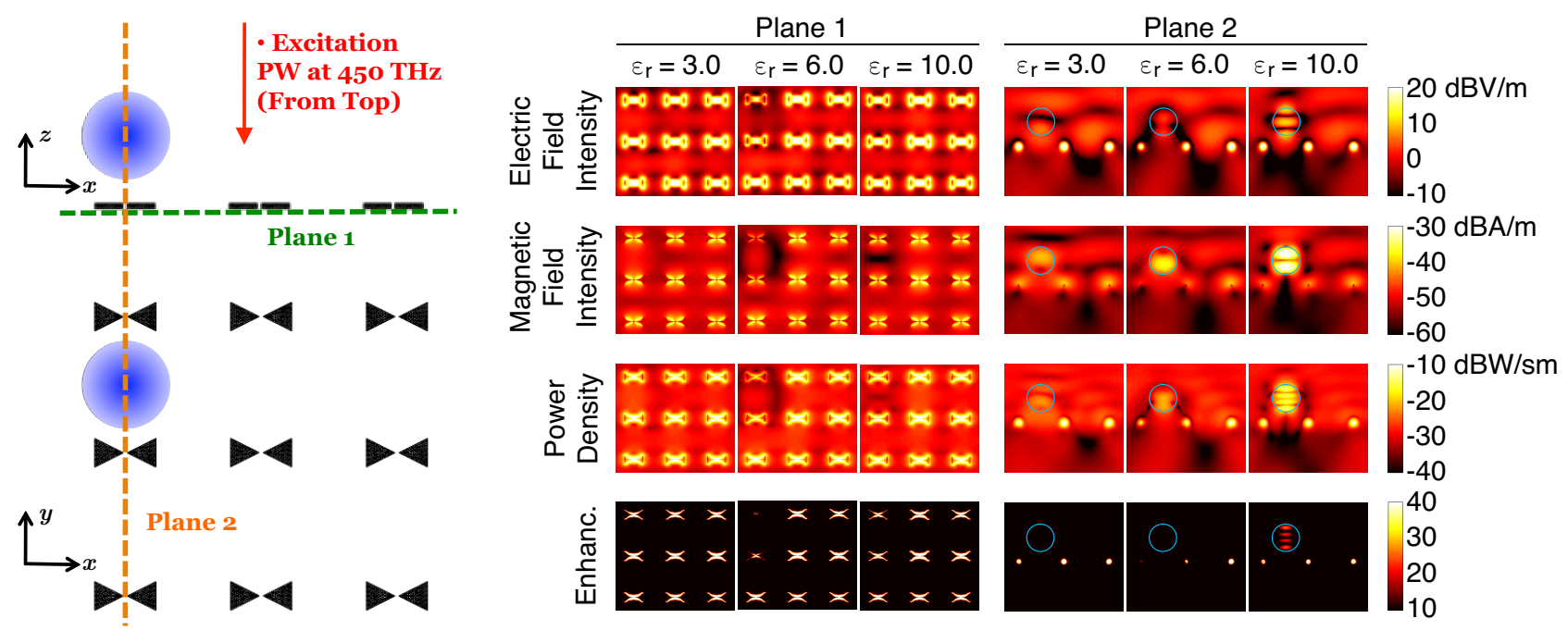

Figure 11: Near-zone characteristics of an array of $3 \times 3$ nanoantennas when dielectric particles (with $\varepsilon=3.0,6.0,10.0$ ) of diameter $300 \mathrm{~nm}$ are located above the array. The periodicity of the array is $475 \mathrm{~nm}$. The particles are $90 \mathrm{~nm}$ away from the top surfaces of the nanoantennas.

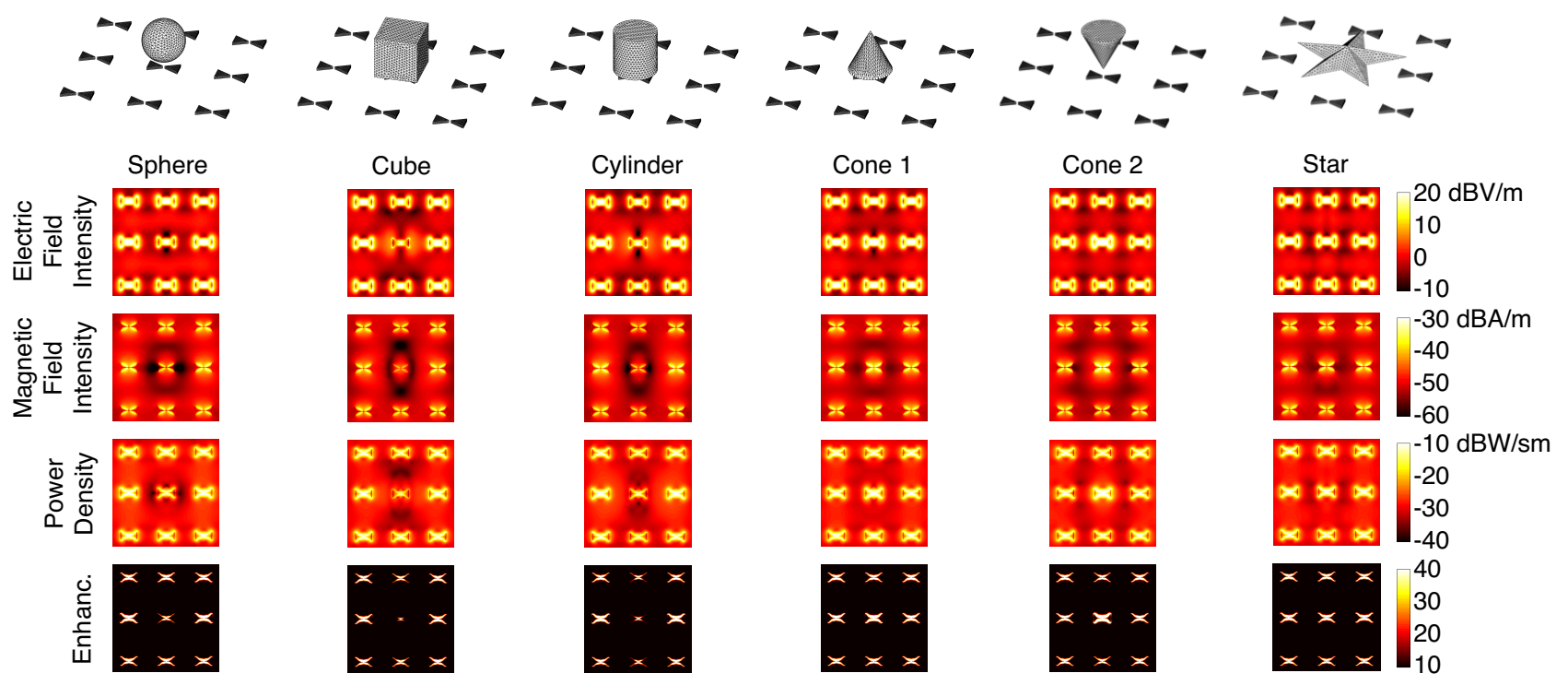

Figure 12: Near-zone characteristics of an array of $3 \times 3$ nanoantennas when dielectric particles with different shapes are located above the array. The periodicity of the array is $475 \mathrm{~nm}$. The particles are $50 \mathrm{~nm}$ away from the top surfaces of the nanoantennas, while their relative permittivity is 8.0.

\section{Acknowledgement}

This work was supported by the Scientific and Technical Research Council of Turkey (TUBITAK) under the Research Grant 118E243 and by the Turkish Academy of Sciences (TUBA).

\section{References}

[1] S.A. Maier, Plasmonics: fundamentals and applications, Springer, 2007.

[2] J. Alda, J.M. Rico-Garcia, J.M. Lopez-Alonso, G. Boreman, Optical antennas for nano-photonic applications, Nanotechnology 16: 230-234, 2005.
[3] Y. Nishijima, L. Rosa, S. Juodkazis, Surface plasmon resonances in periodic and random patterns of gold nano-disks for broadband light harvesting, Opt. Exp. 20: 11466-11477, 2012.

[4] Y.M. El-Toukhy, M. Hussein, M.F.O. Hameed, A.M. Heikal, M.M. Abdelrazzak, S.S.A. Obayya, Optimized tapered dipole nanoantenna as efficient energy harvester, Opt. Exp. 24: 1107-1122, 2016.

[5] T. Kosako, Y. Kadoya, H.F. Hofmann, Directional control of light by a nano-optical yagi-uda antenna, Nat. Photonics 4: 312-215, 2010. 
[6] E.G. Mironov, A. Khaleque, L. Liu, I.S.Maksymov, H.T. Hattori, Enhancing weak optical signals using a plasmonic yagi-uda nanoantenna array, IEEE Photon. Technol. Lett. 26: 2236-2239, 2014.

[7] D.M. Solis, J.M. Taboada, F. Obelleiro, L. Landesa, Optimization of an optical wireless nanolink using directive nanoantennas, Opt. Exp. 21: 2369-2377, 2013.

[8] Y. Yang, Q. Li, M. Qiu, Broadband nanophotonic wireless links and networks using on-chip integrated plasmonic antennas, Sci. Rep. 6: 19490, 2016.

[9] A. Kinkhabwala, Z. Yu, S. Fan, Y. Avlasevich, K. Müllen, W.E. Moerner, Large single-molecule fluorescence enhancements produced by a bowtie nanoantenna, Nat. Photonics 3: 654-657, 2009.

[10] T. Lohmüller, L. Iversen, M. Schmidt, C. Rhodes, H.L. Tu, W.-C. Lin, J.T. Groves, Single molecule tracking on supported membranes with arrays of optical nanoantennas, Nano Lett. 12: 1717-1721, 2012.

[11] K.B. Crozier, W. Zhu, D.Wang, S. Lin, M.D. Best, J.P. Camden, Plasmonics for surface enhanced raman scattering: nanoantennas for single molecules, IEEE J. Sel. Top. Quantum Electron. 20: 3, 2014.

[12] M. Alavirad, L. Roy, P. Berini, Optimization of plasmonics nanodipole antenna array for sensing applications, IEEE J. Sel. Top. Quantum Electron. 20: 3, 2014.

[13] D.P. Fromm, A. Sundaramurthy, P.J. Schuck, G.S. Kino, W.E. Moerner, Gap-dependent optical coupling of single bowtie nanoantennas resonant in the visible, Nano Lett. 4: 957-961, 2004.
[14] E. Üstün, Ö. Eroğlu, U.M. Gür, and Ö. Ergül, Investigation of nanoantenna geometries for maximum field enhancements at optical frequencies, Proc. Progress in Electromagnetics Research Symp. (PIERS), pp. 3673-3680, 2017.

[15] B. Karaosmanoğlu, A. Y1lmaz, Ö. Ergül, A comparative study of surface integral equations for accurate and efficient analysis of plasmonic structures, IEEE Trans. Antennas Propag. 65: 3049-3057, 2017.

[16] A. Çekinmez, B. Karaosmanoğlu, Ö. Ergül, Integralequation formulations of plasmonic problems in the visible spectrum and beyond," Dynamical Systems - Analytical and Computational Techniques, InTech, 2017.

[17] P.B. Johnson, R.W. Christy, Optical constants of the noble metals, Phys. Rev. B 6: 4370-4379, 1972.

[18] Ö. Ergül, L. Gürel, The Multilevel Fast Multipole Algorithm (MLFMA) for Solving Large-Scale Computational Electromagnetics Problems, WileyIEEE, 2014

[19] B. Karaosmanoğlu, A. Y1lmaz, U.M. Gür, Ö. Ergül, Solutions of plasmonic nanostructures using the multilevel fast multipole algorithm, Int. J. RF Microwave Comput.-Aided. Eng. 26: 335-341, 2016. 